\title{
CALIDAD DE VIDA RELACIONADA CON LA SALUD Y VARIABLES PSICOSOCIALES: CARACTERIZACIÓN DE UNA MUESTRA DE MUJERES URUGUAYAS CON CÁNCER DE MAMA
}

\author{
Micaela Reich' y Eduardo Remor ${ }^{2}$ \\ 1 Universidad Católica del Uruguay, Uruguay \\ 2 Universidad Autónoma de Madrid, España
}

\section{Resumen}

La calidad de vida relacionada con la salud (CVRS) es un constructo complejo y multidimensional que puede verse afectado por diferentes aspectos, tales como, sociodemográficos, laborales, clínicos, y psicosociales. El objetivo del presente estudio fue examinar la relación existente entre el estrés percibido, las estrategias de afrontamiento, y la sintomatología depresiva, y su efecto sobre la CVRS. Se estudió una muestra de 116 mujeres uruguayas diagnosticadas e intervenidas quirúrgicamente por cáncer de mama, que acudían a consulta externa tanto en centros de salud de la esfera pública, como privada. Se determinaron aquellas variables asociadas con un mejor ajuste a la enfermedad y sus tratamientos, y una mejor calidad de vida. Se evaluó los niveles de estrés percibido (PSS) y sintomatología depresiva (BDI-II), las estrategias de afrontamiento (Brief COPE), y la CVRS (MOSSF-36) en una única toma de medidas por medio de cuestionarios de auto-informe. Los resultados indicaron la relación de una serie de indicadores sociodemográficos, clínicos y psicológicos con la CVRS actual de las mujeres evaluadas. El análisis de los datos, a través de ANOVA, indicó que la cirugía con posterior reconstrucción mamaria se encontró asociada a niveles significativamente menores de estrés percibido y síntomas depresivos, y a una mejor CVRS.

Palabras Clave: Cáncer de mama, aspectos psicológicos, calidad de vida relacionada con salud, estrés, depresión, afrontamiento.

\section{Abstract}

Health related quality of life (HRQOL) is a complex multidimensional construct that may be affected by demographic, medical, and psychosocial variables. The aim of this study was to examine the association between perceived stress, coping strategies, and depressive symptomatology, and HRQOL outcomes. Participants included 116 Uruguayan women diagnosed with breast cancer who underwent surgery and who were attending ambulatory follow-ups either in private or public health care centers. Factors associated with better adjustment to the disease and its treatment and with higher levels of quality of life, were identified and highlighted. Perceived stress (PSS), depressive symptoms (BDI II), coping strategies (Brief COPE), and HRQOL (MOSSF-36) were assessed utilizing self-report questionnaires. The results yielded several statistically significant associations between demographic, medical, and psychological factors and participant's HRQOL. ANOVA analyses indicated surgery for breast cancer with reconstruction was associated to significantly lower levels of perceived stress and depressive symptoms, and to a better HRQOL.

Keywords: Breast cancer, psychological aspects, health related quality of life, stress, depression, coping.

\section{Correspondencia:}




\section{INTRODUCCIÓN}

El cáncer de mama es el tumor mundialmente más frecuente ${ }^{(1)}$. Se estima que en el mundo cada año un total aproximado de un 1,1 millones de mujeres son diagnosticadas de cáncer de mama, representando un $10 \%$ del total de nuevos diagnósticos oncológicos ${ }^{(2)}$. El colectivo de mujeres con cáncer de mama integra el grupo de sobrevivientes oncológicas de género femenino más numeroso ${ }^{(3)}$. $\mathrm{El}$ creciente número a nivel internacional de sobrevivientes de cáncer de mama refleja el aumento en las tasas de incidencia de la enfermedad, el diagnóstico de la misma mayormente en estadios iniciales que permite mejor evolución y pronóstico, y la adopción generalizada de tratamientos adyuvantes efectivos.

En el Uruguay el cáncer de mama es el de mayor incidencia en la mujer uruguaya y constituye la primera causa de muerte por cáncer en la mujer (p.108) $)^{(4)}$. Los más recientes datos de la Comisión Honoraria de Lucha Contra el Cáncer ${ }^{(5)}$ indican que anualmente se registran a nivel nacional 1.800 nuevos casos de cáncer de mama y aproximadamente 630 mujeres mueren por año a causa de esta enfermedad ${ }^{(6)}$.

La literatura internacional en el tema señala diversas variables sociodemográficas, psicosociales y de carácter clínico como relevantes en el ajuste a la enfermedad y sus tratamientos, y la Calidad de Vida Relacionada con la Salud (en adelante CVRS) de estas mujeres; algunas de ellas, abordadas en este estudio. Por ejemplo, Wapnir et al..$^{(7)}$ encontraron que las mujeres que habían sido intervenidas con cirugía conservadora tenían mejor CVRS que las mujeres mastectomizadas sin reconstrucción. Asimismo, la cirugía conservadora se encontró asociada a mejor capacidad para llevar a cabo actividades de vida diaria ${ }^{(8)}$. Por otro lado, las mujeres mastectomizadas con reconstrucción mamaria presentaron mejor salud mental y funcionamiento físico que aquellas sin reconstrucción ${ }^{(9)}$.

Por otro lado, Holland ${ }^{(10)}$ describió que a mayor tiempo transcurrido desde el diagnóstico de cáncer de mama se predecía mejor CVRS.

Se ha encontrado que los niveles de depresión altos son predictores de una menor $\mathrm{CVRS}^{(11-13)}$, y se asocian a mayores niveles de estrés ${ }^{(14,15)}$ y a menor tiempo transcurrido desde el diagnóstico de cáncer de mama $^{(10)}$.

Carver et al. ${ }^{(16)}$ encontraron que las mujeres que usaban estrategias de afrontamiento caracterizadas por focalizarse en la solución de los problemas, como el optimismo, humor, y búsqueda de apoyo social, presentaban mejores niveles de ajuste al cáncer de mama que las mujeres que no usaban esas estrategias de afrontamiento. Las estrategias de afrontamiento de aproximación centradas en el problema se encontraron asociadas a menores niveles de malestar ${ }^{(17)}$. En esta dirección, un estudio conducido por Pieterse et al.(18), encontró que un estilo pasivo de afrontamiento estaba asociado con niveles altos de malestar en mujeres con cáncer de mama. Las estrategias de afrontamiento de tipo evitativo se encontraron vinculadas a menores niveles de calidad de vida mental de estas mujeres ${ }^{(19)}$. Asimismo, el estilo de afrontamiento a través de la desvinculación, predijo mayores niveles de malestar ${ }^{(20)} ; \mathrm{y}$ mayores niveles de malestar predijeron peor $\mathrm{CVRS}^{(21)}$. A pesar de que los hallazgos no son consistentemente concluyentes, el cuerpo de literatura sobre el tema sugiere que el afrontamiento por medio de estrategias evitativas incide en detrimento del ajuste a largo plazo, mientras que las estrategias de afrontamiento como la aceptación, búsqueda de apoyo social, expresión de emociones, y las estrategias de afrontamiento por aproximación predicen menores niveles de malestar ${ }^{(16,17,22,23)}$. 
Dada la amplia variabilidad observada en términos de ajuste a la enfermedad ${ }^{(24-26)}$, la caracterización de aquellos factores contextuales o personales que facilitan un mejor ajuste y adaptación psicosocial representa un importante cometido para la investigación en cáncer de mama.

Una considerable proporción de la literatura en cáncer de mama ha estudiado una única variable psicosocial en forma aislada, y los efectos de esta sobre la CVRS de las mujeres. Las pacientes a menudo experimentan sintomatología diversa. La concurrencia de sintomatología podría conllevar efectos adversos en el bienestar de estas mujeres. La CVRS está compuesta de diversos aspectos que pueden verse afectados por factores sociodemográficos, médicos, y psicosociales ${ }^{(27)}$. En contraste con el limitado efecto de las variables médicas iniciales recogido en la literatura, se observan evidencias emergentes de que las variables psicosociales juegan un papel fundamental en la predicción de aspectos emocionales de la CVRS ${ }^{(27)}$. Considerando la ausencia de información en el contexto uruguayo, ha surgido el interés en conocer la CVRS y las variables psicosociales asociadas a la misma; sea a través de la comparación de dichos factores respecto de las mujeres saludables (entendiendo en este caso al colectivo como población relativamente homogénea), sea comparando subgrupos en nuestra muestra de mujeres con cáncer de mama, partiendo de la base de que presenta características diferentes según difieran determinadas variables sociodemográficas, laborales, psicosociales, o clínicas.

En el presente estudio se describen aspectos sociodemográficos, laborales, clínicos, psicológicos y de la CVRS de una muestra de mujeres uruguayas diagnosticadas con cáncer de mama, que recibían atención médica post-quirúrgica en centros de salud del ámbito público y privado a nivel nacional. Se trata de identificar las variables asociadas con un mejor ajuste a la enfermedad, así como con una mejor CVRS, a través de la identificación de los factores sociodemográficos, laborales y clínicos vinculados a las áreas de CVRS y salud mental.

\section{MÉTODO}

\section{Participantes}

La muestra de participantes de este estudio estuvo compuesta por 116 mujeres uruguayas con cáncer de mama postcirugía, que se encontraban próximas al período de inicio, durante, o bien habiendo finalizado los tratamientos coadyuvantes (p. ej., radioterapia y/o quimioterapia) en diferentes estadios de la enfermedad: I, II, III y IV de acuerdo a clasificación clínica del Cáncer de Mama ${ }^{(28)}$ y se encontraban recibiendo asistencia en centros de salud de Uruguay, tanto de la esfera pública como privada. Las participantes fueron reclutadas a través de un muestreo no-probabilístico, por conveniencia.

Para formar parte del presente estudio fueron considerados criterios de inclusión: (i) que haya transcurrido más de un mes desde el momento del diagnóstico; (ii) que al momento del estudio la participante haya tenido su primer cirugía para el tratamiento de la enfermedad (pudiendo tratarse de cualquiera de los procedimientos quirúrgicos protocolares disponibles para tratar el cáncer de mama); (iii) participación voluntaria y anónima; (iv) nivel cultural que habilite a la comprensión y cumplimentación de los cuestionarios; (v) asistencia a consulta ambulatoria en centros de salud de Uruguay; (vi) no tener una historia de patología neoplásica anterior.

Han sido considerados criterios de exclusión: (i) estar consumiendo medicación psicoactiva; (ii) presentar cualquier desorden mental o cognitivo que pudiera dificultar o impedir cumplimentar los cuestio- 
narios de autoinforme (el cuestionario de datos clínicos diseñado para el presente estudio incluye preguntas respecto a diagnóstico y tratamiento de salud mental recibidos); (iii) haber sido diagnosticada de otra patología grave o incapacitante aparte del cáncer.

A los efectos de evaluar los criterios de inclusión y exclusión mencionados se utilizó el formulario de datos sociodemográficos, laborales y clínicos diseñado ad hoc para este estudio. A través del mismo, así como de la previa revisión de Historias Clínicas, se excluyó de la muestra a las participantes que no cumplieran con los criterios descritos.

\section{Instrumentos de Medida}

Entre paréntesis se indica el valor de Alpha de Cronbach de los distintos instrumentos de medida, así como de las subescalas y Componentes a partir de su administración en la muestra de participantes del presente estudio.

Cuestionario de Salud MOS-SF-36 versión $2.0^{(29,30)}$ en su versión adaptada para Uruguay (http://www.iqola.org), que evalúa la calidad de vida relacionada con la salud en poblaciones adultas (mayores de 16 años). Se trata de un instrumento de autoinforme que evalúa la CVRS, y que consta de 36 ítems con un formato de respuesta de una escala tipo Likert. Las puntuaciones directas se traducen a puntuaciones escalares que pueden tomar un valor de 0 a 100. Una puntuación mayor indica mayor CVRS ( $\alpha$ de Cronbach de la escala global $=$,95). El producto de su aplicación es la construcción de 8 dimensiones o escalas de salud, resultado del promedio de la suma de las preguntas contenidas en cada escala. Estos conceptos son: a) Función Física (FF) $(\alpha=, 92)$, b) Rol Físico (RF) $(\alpha=, 94), \mathrm{c})$ Dolor Corporal (DC) $(\alpha$ $=, 87)$, d) Salud General (SG) $(\alpha=, 81)$, e) Vitalidad (VT) $(\alpha=, 75)$, f) Función Social
(FS) $(\alpha=, 62)$, g) Rol Emocional (RE) ( $\alpha=$ ,93), y h) Salud Mental (SM) $(\alpha=, 81)$, Las mencionadas subescalas se agrupan en los Componentes de Salud Físico $(\alpha=, 93)$, y Mental $(\alpha=90)$, Además de estas dimensiones de salud, el MOS-SF-36 incluye el concepto general de cambios en la percepción del estado de salud actual y en la del año anterior.

Inventario de Depresión de Beck II (BDI II $\left.^{(31)}\right)$, versión en español(32), un inventario de autoinforme de 21 ítems frecuentemente empleado para evaluar síntomas depresivos a nivel cognitivo, afectivo y vegetativo. En su estructura se destaca una construcción que refiere a grados de intensidad de la sintomatología, en frases que van de la menor a la mayor gravedad (0 a 3 puntos) $(\alpha$ de Cronbach $=, 90)$.

Brief COPE ${ }^{(33)}$, en su versión en español ${ }^{(34)}$. Se trata de un instrumento de autoinforme que evalúa las estrategias de afrontamiento empleadas, y que consta de 24 ítems con un formato de respuesta de una escala tipo Likert de 4 puntos que va de 1 "no hice esto en lo absoluto" a 4 "hice esto con mucha frecuencia". Brief COPE-versión castellano está compuesto por las siguientes escalas: a) Auto-distracción $(\alpha=, 58)$, b) Afrontamiento activo ( $\alpha$ $=, 84)$, c) Negación $(\alpha=, 52)$, d) Uso de sustancias $(\alpha=, 83)$, e) Utilización de apoyo emocional ( $\alpha=, 84)$, f) Desvinculación comportamental $(\alpha=, 66)$, g) Expresión de emociones negativas $(\alpha=, 57)$, h) Reinterpretación positiva $(\alpha=, 76)$, i) Planificación $(\alpha=, 65), \mathrm{j})$ Humor $(\alpha=, 80), \mathrm{k})$ Aceptación $(\alpha=, 70)$, y l) Religión ( $\alpha=$ ,90), El $\alpha$ de Cronbach global de la escala fue de .84 .

Escala de Estrés Percibido (PSS) ${ }^{(35)}$, en su versión 2.0 en español ${ }^{(36)}$. La PSS fue diseñada para medir el grado en que las situaciones en la vida se valoran como estresantes. Se trata de un instrumento de autoinforme que evalúa el nivel de estrés percibido durante el último mes, y que consta 
de 14 ítems con un formato de respuesta de una escala tipo Likert de 5 puntos que va de 0 "nunca" a 4 "muy a menudo" $(\alpha$ de Cronbach $=, 86$ ).

Encuesta de datos sociodemográficos, laborales, y clínicos (elaborada ad hoc para este estudio), incluye información respecto a: tiempo transcurrido desde el diagnóstico de cáncer de mama, status clínico, tratamientos recibidos por el diagnóstico de cáncer de mama, diagnósticos neoplásicos anteriores, otras enfermedades graves además de cáncer de mama, antecedentes neoplásicos familiares, estadio de extensión de la enfermedad, consulta a un profesional de la salud mental, diagnóstico de trastorno de salud mental, tiempo transcurrido desde el diagnóstico de trastorno de salud mental, tratamiento psicológico o psiquiátrico, edad, lugar de residencia, estado civil, personas con las que convive, existencia de hijos, profesión, situación laboral, estudios formales más avanzados completados, creencia religiosa (incluye una pregunta respecto a si la participante es o no creyente, y a la religión con la que se identifica en caso de serlo).

\section{Procedimientos}

Antes del inicio del estudio, el proyecto de investigación fue evaluado y aprobado por el Comité de Ética de Investigación de la Universidad Católica del Uruguay (UCUDAL); asimismo se ha obtenido la aprobación de los Comités de Ética y Bioética y el cuerpo médico responsable de los Departamentos de Oncología de cada Institución que colaboró en el proyecto. La administración del protocolo de evaluación se ha llevado a cabo por un profesional investigador y estudiantes avanzados de la Licenciatura en Psicología de UCUDAL previamente entrenados para la tarea. La selección de las participantes se llevó a cabo de forma consecutiva, contactando a las mismas en el momento en que salieran, o se encontraran en sala de espera de consulta con su médico tratante (previa revisión de Historia Clínica). Se les explicaron los aspectos relevantes del estudio científico y se les solicitó el consentimiento informado. Una vez que las mujeres manifestaban por escrito su participación voluntaria, se realizó una única evaluación mediante cuestionarios de autoinforme (completados por las pacientes-participantes). Se contrabalanceó sistemáticamente el orden en que se presentaban las medidas de las variables estudiadas, a fin de reducir los efectos del orden ${ }^{(37)}$. El tiempo aproximado de evaluación por participante fue de entre 30-45 minutos.

\section{ANÁLISIS ESTADÍSTICO}

Para Ilevar a cabo el análisis estadístico de los datos se utilizó el paquete estadístico SPSS versión 18.0 para Windows. Los datos recabados fueron revisados por los investigadores para minimizar los errores de transcripción de la información a la base de datos. Inicialmente se condujeron análisis de frecuencias y análisis descriptivos con el objetivo de representar los datos. De las escalas utilizadas, se obtuvieron estadísticos de normalidad, asimetría y curtosis. Como medidas de resumen se calcularon las medias, mínimos y máximos, y desviaciones típicas. Se condujo un análisis de correlación de Pearson para determinar el grado de asociación lineal entre las variables de intervalo y ordinales con distribución normal, y el coeficiente de correlación de Spearman para determinar el grado de asociación lineal entre las variables de intervalo y ordinales que no presentaron distribución normal. Éstas últimas debieron ser transformadas en rangos para su análisis. Adicionalmente, se llevó a cabo un análisis de contingencias para poner a prueba la independencia entre las variables dicotómicas o nominales. Para valorar las diferencias entre grupos en 
relación a las variables sociodemográficas, laborales, clínicas, psicosociales y la CVRS se utilizó un análisis de varianza de un factor, la prueba $F$ del ANOVA Paramétrico o el ANOVA no paramétrico de KruskallWallis (para el caso de más de dos grupos), y la prueba $U$ de Mann-Whitney de acuerdo al número de comparaciones a analizar. Para reducir la probabilidad de error Tipo l, se llevó a cabo el ajuste de Bonferroni. Para todos los procedimientos estadísticos se tomó un valor de significación de $p<, 05$.

\section{RESULTADOS}

En el presente apartado se exponen los principales resultados alcanzados a partir del estudio, buscando dar respuesta a los objetivos anteriormente descritos. Las Tablas 1 y 2 resumen la información referida a las características sociodemográficas y de salud de la muestra estudiada.

Puede observarse en la Tabla 1 que las participantes de este estudio eran mayoritariamente mayores de 40 años, residían en zonas urbanas, se encontraban casadas

Tabla 1. Características sociodemográficas y laborales más salientes $(n=116)$; Uruguay

\begin{tabular}{|c|c|c|c|}
\hline & $\mathbf{M}$ & DT & $\%$ \\
\hline Edad (años) & 50,79 & 9,395 & \\
\hline $\begin{array}{l}\text { Lugar de residencia: } \\
\text { Capital } \\
\text { Ciudad pequeña } \\
\text { Otras ciudades pequeñas o pueblos }\end{array}$ & & & $\begin{array}{c}50 \\
33,6 \\
16,4\end{array}$ \\
\hline $\begin{array}{l}\text { Estado civil: } \\
\text { soltera } \\
\text { divorciada } \\
\text { casada, en pareja de hecho } \\
\text { Viuda }\end{array}$ & & & $\begin{array}{l}6,0 \\
22,4 \\
58,7 \\
12,9\end{array}$ \\
\hline $\begin{array}{l}\text { Situación laboral: } \\
\text { laboralmente activa } \\
\text { ama de casa } \\
\text { incapacidad laboral transitoria } \\
\text { jubiladas, pensionistas }\end{array}$ & & & $\begin{array}{l}55,2 \\
33,6 \\
13,8 \\
26,7\end{array}$ \\
\hline $\begin{array}{l}\text { Convivencia: } \\
\text { vive en pareja (exclusivamente) } \\
\text { vive con hijos (exclusivamente) } \\
\text { vive en pareja y con al menos un } \\
\text { hijo } \\
\text { vive sola } \\
\text { vive con amigos o hermanos }\end{array}$ & & & $\begin{array}{r}16,4 \\
20,7 \\
42,2 \\
17,2 \\
3,4 \\
\end{array}$ \\
\hline $\begin{array}{l}\text { Nivel educativo: } \\
\text { Sin estudios } \\
\text { Primaria } \\
\text { Secundaria o bachillerato } \\
\text { Universitarios }\end{array}$ & & & $\begin{array}{c}1,7 \\
47,4 \\
21,6 \\
29,3\end{array}$ \\
\hline
\end{tabular}


Tabla 2. Características de Salud más salientes $(n=116)$; Uruguay

\begin{tabular}{|c|c|c|}
\hline & $\mathbf{M}$ & $\%$ \\
\hline $\begin{array}{l}\text { Tiempo transcurrido desde el diagnóstico (años): } \\
<1 \text { año } \\
2-5 \text { años } \\
>5 \text { años }\end{array}$ & 4.47 & $\begin{array}{c}24.2 \\
34.5 \\
26 \\
\end{array}$ \\
\hline $\begin{array}{l}\text { Tiempo hospitalizada en último año: } \\
<1 \text { semana } \\
1-3 \text { meses } \\
\text { No estuvo hospitalizada } \\
\end{array}$ & & $\begin{array}{c}80 \\
10.3 \\
9.7 \\
\end{array}$ \\
\hline $\begin{array}{l}\text { Status clínico: } \\
\text { en remisión completa } \\
\text { en tratamiento adyuvante } \\
\text { En remisión parcial } \\
\end{array}$ & & $\begin{array}{c}51 \\
40 \\
9\end{array}$ \\
\hline $\begin{array}{l}\text { Tipo de cirugía: } \\
\text { mastectomía radical modificada s/reconstrucción } \\
\text { mastectomía radical modificada c/reconstrucción } \\
\text { Cirugía conservadora }\end{array}$ & & $\begin{array}{c}40.5 \\
25.9 \\
33\end{array}$ \\
\hline $\begin{array}{l}\text { Tratamientos adyuvantes: } \\
\text { quimioterapia } \\
\text { radioterapia } \\
\text { hormonoterapia } \\
\text { quimioterapia y radioterapia } \\
\text { quimioterapia, radioterapia, y hormonoterapia } \\
\text { únicamente quimioterapia y radioterapia } \\
\text { quimioterapia, pero no radioterapia } \\
\text { únicamente quimioterapia y hormonoterapia } \\
\text { únicamente hormonoterapia } \\
\text { únicamente quimioterapia } \\
\text { únicamente radioterapia } \\
\text { Sin tratamiento adyuvante }\end{array}$ & & $\begin{array}{c}82.8 \\
62.1 \\
55.2 \\
57 \\
53.4 \\
43 \\
26 \\
22 \\
7 \\
4.3 \\
5 \\
5 \\
\end{array}$ \\
\hline $\begin{array}{l}\text { Antecedentes oncológicos familiares: } \\
\text { sin antecedentes } \\
\text { abuelos } \\
\text { madre } \\
\text { padre } \\
\text { hermano/s } \\
\text { otros familiares }\end{array}$ & & $\begin{array}{c}7 \\
33.6 \\
21.6 \\
12 \\
8.6 \\
17.2 \\
\end{array}$ \\
\hline $\begin{array}{l}\text { Estadio: } \\
\text { I o II } \\
\text { III } \\
\text { IV } \\
\end{array}$ & & $\begin{array}{c}77.6 \\
19 \\
3.4\end{array}$ \\
\hline $\begin{array}{l}\text { Consulta a profesional de salud mental: } \\
\mathrm{Si} \\
\text { Motivo de la consulta: } \\
\text { debido a problemas vinculados al cáncer de mama } \\
\text { otros motivos } \\
\text { Tiempo de la consulta: } \\
<2 \text { años previo a la evaluación } \\
2-5 \text { años previo a la evaluación } \\
>5 \text { años previo a la evaluación } \\
\end{array}$ & & $\begin{array}{c}59.5 \\
71 \\
28.9 \\
53.6 \\
36 \\
10.4 \\
\end{array}$ \\
\hline $\begin{array}{l}\text { Tratamiento de salud mental: } \\
\mathrm{Si} \\
\text { No } \\
\text { Tipo de tratamiento de salud mental: } \\
\text { tratamiento psicológico individual (no farmacológico) } \\
\text { Tiempo del tratamiento psicológico: } \\
\text { < } 2 \text { años previo a la evaluación } \\
2-5 \text { años previo a la evaluación }\end{array}$ & & $\begin{array}{r}52.6 \\
47.4 \\
95.5 \\
53.7 \\
35.8\end{array}$ \\
\hline
\end{tabular}


Tabla 3. Medias y desviaciones típicas de los instrumentos utilizados ( $\mathbf{n}=116$ ); Uruguay

\begin{tabular}{|l|c|c|}
\hline & $M$ & $D T$ \\
\hline MOS-SF-36 versión 2.0 (Calidad de Vida Relacionada con la salud) & 77,78 & 12,05 \\
Funcionamiento Físico & 73,97 & 25,18 \\
Rol Físico & 66,79 & 25,29 \\
Dolor Corporal & 63,02 & 23,04 \\
Salud General & 57,97 & 19,60 \\
Vitalidad & 54,74 & 16,44 \\
Funcionamiento Social & 70,72 & 23,07 \\
Rol Emocional & 67,62 & 24,81 \\
Salud Mental & 48,36 & 20,21 \\
CSF (Resumen salud física) & 48,13 & 9,03 \\
CSM (Resumen salud mental) & 38,81 & 9,78 \\
BDI II (Depresión) & 12,88 & 9,58 \\
Brief Cope (Afrontamiento) & 2,31 & 0,39 \\
PSS (Estrés) & 22,54 & 8,05 \\
\hline
\end{tabular}

o conviviendo en pareja, y convivían con hijos; la mayoría se encontraban laboralmente activas, y habían completado un nivel de estudios de bachillerato, técnicos, o terciarios. En la Tabla 2 puede observarse que mayoritariamente, las mujeres con cáncer de mama que participaron en este estudio habían sido diagnosticadas 2-5 años previo al momento del estudio, en estadios iniciales de la enfermedad (I y II), y se encontraban en remisión completa. El tipo de cirugía más frecuente entre las participantes fue mastectomía radical modificada sin reconstrucción, y casi el total de mujeres había recibido quimioterapia, radioterapia, o la combinación de ambos tratamientos adyuvantes. Por otro lado, más de la mitad de participantes había recibido tratamiento psicológico menos de 2 años previo al momento del estudio por dificultades vinculadas a la enfermedad o sus tratamientos.

\section{Análisis descriptivo de las escalas utilizadas}

Se presentan los resultados descriptivos de las puntuaciones en las escalas utiliza- das en este estudio (Ver tabla 3). Puede observarse que, entre las subescalas que componen el MOS-SF-36, Funcionamiento Físico es la que presentó puntuaciones que indicaban mejor CVRS, mientras que la subescala de Salud Mental fue en la que las mujeres participantes del presente estudio informaron niveles más bajo de CVRS. Asimismo, puede verse que las mujeres informaron niveles más altos de CVRS en su Componente Físico que en su Componente Mental. Los valores de alpha de Cronbach para las escalas y subescalas se presentan en el apartado de Instrumentos de Medida.

\section{Asociaciones de los indicadores sociodemográficos, clínicos y psicológicos con la CVRS}

Para el análisis correlacional de las variables continuas estudiadas se utilizó el coeficiente Rho de Spearman, o bien un análisis de correlación de Pearson, en virtud del tipo de variable en cuestión. A continuación se presentan los resultados y se comentan los aspectos más salientes de dichos análisis. 
Como se puede identificar en la Tabla 4, se encontraron correlaciones significativas entre diferentes variables. Por ejemplo, la edad $(r=-0,184)$, y la educación ( $r=$ $-0,209)$ han correlacionado negativamente con el Componente de Salud Física (MOSSF-36), indicando que las mujeres más jóvenes y con más educación formal fueron quienes informaron de mejor CVRS física. La educación ha corrrelacionado positivamente con el Componente de Salud Mental (MOS-SF-36) $(r=0,195)$, indicando que las mujeres con mayor nivel educativo informaron de mejores niveles de salud mental. La edad ha correlacionado positivamente $(r=0.387)$, y la educación negativamente $(r=-0,323)$, con el número de ciclos de radioterapia recibidos, indicando que las mujeres mayores y con menores niveles de educación formal fueron quienes recibieron mayor cantidad de ciclos de este tratamiento.

Los Componentes de Salud Mental y Física de la CVRS se han correlacionado positivamente $(r=0,614)$, indicando que las mujeres que informaron de buena salud física también lo hicieron con relación a la salud mental. El nivel de sintomatología depresiva ha correlacionado negativamente con el CSF de la CVRS ( $r=-0,597)$, así como con el CSM de la CVRS $(r=-0,606)$. Asimismo, los niveles de estrés percibido han correlacionado en forma negativa con el CSF $(r=-0,522)$, y el CSM $(r=-0,616)$ de la CVRS. Esto indica que ante mayores niveles de depresión y estrés percibido, las mujeres informaron peores niveles de CVRS. El CSM de la CVRS ha correlacionado positivamente con el tiempo transcurrido desde la consulta a un profesional de la salud mental ( $r=0,556)$, así como con el tiempo transcurrido desde el inicio de un tratamiento de salud mental $(r=0,553)$, indicando que a mayor tiempo desde el momento de consultar a un profesional e iniciar un tratamiento de salud mental, el CSM informado por las participantes fue mejor.
La sintomatología depresiva ha correlacionado positivamente con la sintomatología de estrés percibido $(r=0,662)$, indicando que las mujeres que informaron de síntomas depresivos también lo hicieron en relación a síntomas de estrés percibido.

El uso de un estilo de afrontamiento focalizado en el problema y de un estilo de afrontamiento focalizado en la emoción han correlacionado positivamente $(r$ $=0,678$ ), señalando que las mujeres que informaron hacer uso de uno de estos estilos, también lo hicieron en relación al otro.

El tiempo transcurrido desde el diagnóstico de cáncer de mama ha correlacionado negativamente con el tiempo de internación durante el último año $(r=-0,596)$, y positivamente con el tiempo transcurrido desde la consulta a un profesional de la salud mental $(r=0,528)$ y desde el inicio de un tratamiento de salud mental $(r=0,565)$, indicando que aquellas mujeres que habían sido diagnosticadas más recientemente informaron haber sido ingresadas durante más tiempo durante el año previo a la evaluación, y habían consultado e iniciado tratamiento con un profesional de la salud mental en un momento más próximo al de la recolección de datos.

El tiempo transcurrido desde la consulta a un profesional de la salud mental ha correlacionado positivamente con el tiempo transcurrido desde el inicio de un tratamiento de salud mental $(r=0,955)$ indicando que las mujeres que habían consultado más tiempo previo a la recolección de datos, también habían iniciado un tratamiento en un momento más alejado del de la evaluación. La edad ha correlacionado negativamente con el estilo de afrontamiento focalizado en el problema $(r=-0,253)$, y en la emoción $(r=-0,295)$, implicando que las mujeres más jóvenes fueron quienes hicieron mayor uso de estos estilos de afrontamiento. La educación ha correlacionado positivamente con el 


\begin{tabular}{|c|c|c|c|c|c|c|c|c|c|c|c|c|c|c|c|c|c|c|c|c|c|}
\hline$\stackrel{\theta}{=}$ & & $\underset{1}{*}$ & \begin{tabular}{l}
$\frac{\pi}{2}$ \\
$\stackrel{2}{\sigma}$ \\
\hdashline
\end{tabular} & & $\tilde{D}_{i}^{+}$ & $\overline{8}$ & & $\begin{array}{l}\bar{*} \\
\stackrel{*}{*} \\
\stackrel{2}{\check{N}}\end{array}$ & 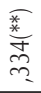 & ర్ & & $\tilde{\delta}_{1}^{2}$ & $\stackrel{L n}{=}$ & $\stackrel{\varrho}{\longleftarrow}$ & $\stackrel{\infty}{\stackrel{\circ}{\curvearrowleft}}$ & 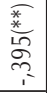 & $\begin{array}{l}\underset{*}{*} \\
\underset{\approx}{7} \\
?\end{array}$ & $\stackrel{\stackrel{m}{m}}{=}$ & & 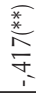 & $\stackrel{8}{\stackrel{-}{-}}$ \\
\hline 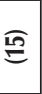 & & $\begin{array}{l}\frac{\pi}{5} \\
\stackrel{5}{0} \\
i\end{array}$ & $\tilde{\tilde{c}}$ & & \&̊․ & 苍 & & 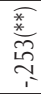 & 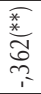 & 8 & & 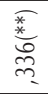 & 番 & 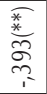 & \begin{tabular}{l} 
悉 \\
\multirow{\sigma}{\pi}{} \\
$?$ \\
$?$
\end{tabular} & $\begin{array}{l}\stackrel{\infty}{\infty} \\
\underset{1}{0}\end{array}$ & 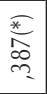 & 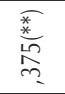 & & $\stackrel{8}{-}$ & \\
\hline 巳 & & 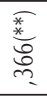 & 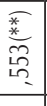 & & 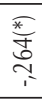 & $\begin{array}{l}\text { 悉 } \\
\stackrel{8}{0} \\
\stackrel{9}{9} \\
i\end{array}$ & & $\stackrel{2}{\circ}$ & \begin{tabular}{l} 
F \\
İ \\
\multirow{2}{*}{}
\end{tabular} & No & & 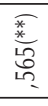 & 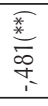 & 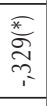 & $\underset{p}{\bar{r}}$ & $\stackrel{\frac{L n}{\tau}}{\underset{N}{2}}$ & $\stackrel{\frac{2 n}{\tau}}{\underset{1}{2}}$ & $\stackrel{8}{8}$ & & & \\
\hline$\stackrel{\bar{m}}{=}$ & & 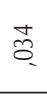 & $\stackrel{\circ}{\circ}$ & & $\stackrel{\infty}{\simeq}$ & $\stackrel{20}{=}$ & & $\stackrel{\text { 导 }}{\square}$ & ఃे & $\stackrel{0}{\varrho}$ & & ָิ & $\begin{array}{l}\frac{\pi}{*} \\
o \% \\
? \\
?\end{array}$ & 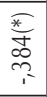 & $\underset{\tau}{\stackrel{m}{r}}$ & 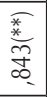 & $\underset{-}{8}$ & & & & \\
\hline$\widehat{\mathbb{E}}$ & & \&̊ㅜㅇ & $\stackrel{20}{=}$ & & 。 & $\stackrel{5}{T}$ & & ฮี & $\underset{\sim}{ָ}$ & 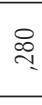 & & $\bar{m}$ & 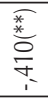 & 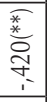 & $\begin{array}{l}\frac{\pi}{*} \\
\stackrel{0}{0} \\
? ? \\
?\end{array}$ & $\underset{8}{8}$ & & & & & \\
\hline$\widehat{E}$ & & $\stackrel{2}{0}$ & 号 & & కి & 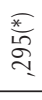 & & $\hat{\tilde{o}}_{1}$ & $\stackrel{\text { Lิ }}{0}$ & : & & $\underset{F}{\mp}$ & 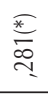 & $\begin{array}{l}\frac{}{*} \\
\frac{1}{0} \\
\stackrel{2}{0} \\
\infty\end{array}$ & $\stackrel{-}{\circ}$ & & & & & & \\
\hline 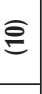 & & 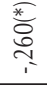 & 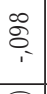 & & $\stackrel{\infty}{\simeq}$ & 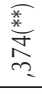 & & $\overbrace{1}^{\circ}$ & $\stackrel{2}{\stackrel{8}{8}}$ & 吉 & & $\begin{array}{l}\frac{\pi}{*} \\
\hat{5} \\
? \\
.\end{array}$ & 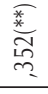 & $\underset{-}{8}$ & & & & & & & \\
\hline ब & & $\begin{array}{l}\underset{*}{*} \\
\underset{0}{*} \\
i\end{array}$ & 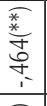 & & $\begin{array}{l}\frac{F}{*} \\
\infty \\
=\end{array}$ & $\begin{array}{l}\text { 悉 } \\
\stackrel{\text { In }}{\pi}\end{array}$ & & $\hat{\sigma}$ & $\stackrel{\nabla}{\sigma}$ & $\hat{\sigma}_{i}$ & & 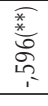 & $\stackrel{8}{\circ}$ & & & & & & & & \\
\hline$\widehat{\Phi}$ & & 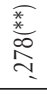 & $\begin{array}{l}\text { F } \\
\text { 孚 } \\
\text {. }\end{array}$ & & $\stackrel{8}{\circ}$ & $\underset{n}{5}$ & & $\stackrel{\infty}{\underset{1}{-1}}$ & $\stackrel{\infty}{0}_{i}^{0}$ & $\frac{g}{\underset{T}{T}}$ & & $\stackrel{8}{8}$ & & & & & & & & & \\
\hline$E$ & & 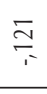 & 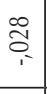 & & $\begin{array}{l}\infty \\
\infty \\
0 \\
1\end{array}$ & $\stackrel{\cong}{\stackrel{\longrightarrow}{\sim}}$ & & 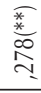 & 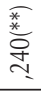 & $\underset{8}{8}$ & & & & & & & & & & & \\
\hline (e) & & 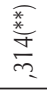 & \begin{tabular}{l}
$\frac{\pi}{*}$ \\
\multirow{2}{*}{} \\
\multirow{2}{*}{}
\end{tabular} & & 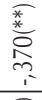 & $\begin{array}{l}\frac{\pi}{*} \\
\stackrel{4}{L} \\
\dddot{m} \\
i\end{array}$ & & $\begin{array}{l}\frac{\pi}{*} \\
\frac{*}{*} \\
0 \\
6 \\
0\end{array}$ & $\stackrel{8}{8}$ & & & & & & & & & & & & \\
\hline$\sqrt{6}$ & & 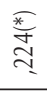 & 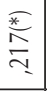 & & $\begin{array}{l}\stackrel{*}{*} \\
\stackrel{n}{m} \\
?\end{array}$ & 悉 & & 8 & & & & & & & & & & & & & \\
\hline 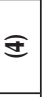 & & 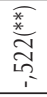 & $\begin{array}{l}\frac{\pi}{2} \\
\frac{2}{6} \\
6 \\
1\end{array}$ & & 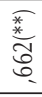 & $\stackrel{8}{\circ}$ & & & & & & & & & & & & & & & \\
\hline त्र & & 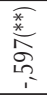 & $\begin{array}{l}\frac{\pi}{2} \\
8 \\
0 \\
0 \\
1\end{array}$ & & 8 & & & & & & & & & & & & & & & & \\
\hline ब & & $\begin{array}{l}\frac{\pi}{*} \\
\frac{7}{6} \\
\end{array}$ & 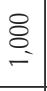 & & & & & & & & & & & & & & & & & & \\
\hline & 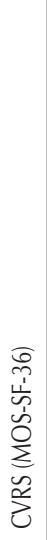 & $\stackrel{\overrightarrow{\tilde{U}}}{\underline{E}}$ & 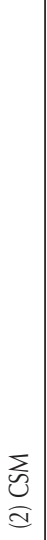 & 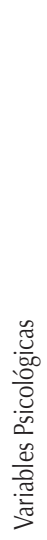 & 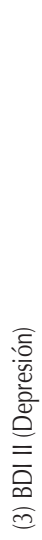 & 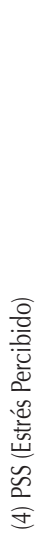 & 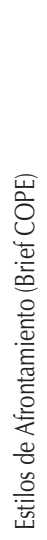 & 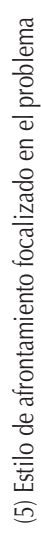 & 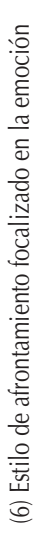 & 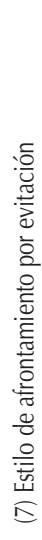 & 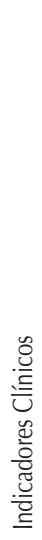 & 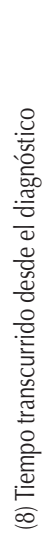 & 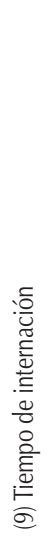 & 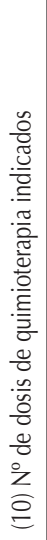 & 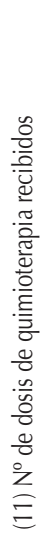 & 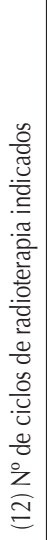 & 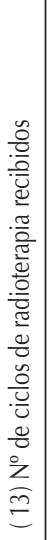 & 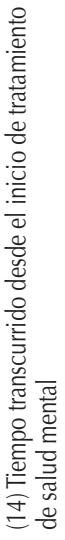 & 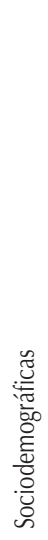 & 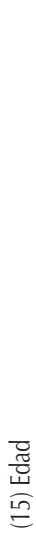 & 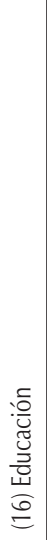 \\
\hline
\end{tabular}


estilo de afrontamiento focalizado en el problema $(r=0,295)$, y en la emoción $(r$ $=0,334)$, implicando que las mujeres más educadas fueron quienes hicieron mayor uso de estos estilos de afrontamiento. La edad ha correlacionado positivamente con el tiempo transcurrido desde el diagnóstico de cáncer de mama $(r=0,336)$, y negativamente con el tiempo de hospitalización durante el año previo a la recogida de datos $(r=-0,344)$, y el número de dosis de quimioterapia indicados $(r=-0,393)$, y recibidos $(r=-0,362)$, indicando que las mujeres más jóvenes fueron quienes habían sido diagnosticadas más recientemente, quienes habían sido hospitalizadas en mayor medida, y a quienes se les había prescripto y suministrado mayor cantidad de dosis de quimioterapia adyuvante. La educación ha correlacionado negativamente con el número de ciclos de radioterapia recibidos $(r=-0,395)$, indicando que las mujeres menos educadas fueron quienes recibieron mayor cantidad de ciclos de este tratamiento. La edad ha correlacionado positivamente con el tiempo transcurrido desde el inicio de un tratamiento de salud mental $(r=0,375)$, y negativamente con la educación $(r=-0,417)$, indicando que las mujeres más jóvenes fueron quienes habían recibido tratamiento psicológico más recientemente, y habían completado niveles más altos de educación formal respecto de las mayores.

Se condujeron análisis adicionales buscando asociaciones entre las variables nominales. Los resultados muestran que el CSF de la CVRS se encontró asociado con la situación laboral $(C=0,496, p=0,000)$, el nivel de estudios alcanzado $(C=0,396$, $p=0,006)$, la fase clínica en que se encontraban las mujeres $(C=0,413, p=0,001)$, y el tipo de cirugía $(C=0,344, p=0,016)$. El CSM de la CVRS se encontró asociado con la situación laboral $(C=0,463, p=$ $0,024)$, el nivel de estudios alcanzado $(C$
$=0,413, p=0,021)$, la fase clínica en que se encontraban las mujeres $(C=0,497, p=$ 0.000), y el tipo de cirugía $(C=0,358, p=$ $0,048)$. La sintomatología depresiva se encontró asociada con la situación laboral $(C$ $=0.554, p=0.000$ ), la fase clínica en que se encontraban las mujeres $(C=0,378, p$ $=0,023)$, el tipo de cirugía $(C=0,412, p$ $=0,005)$, inmunoterapia adyuvante $(C=$ $0,331, p=0,003)$, y el estadio de la enfermedad $(C=0,406, p=0,007)$.

El estilo de afrontamiento focalizado en el problema se encontró asociado con el estado civil $(C=0,669, p=0,009)$, la situación laboral $(C=0,767, p=0,000)$, el nivel de estudios alcanzado $(C=0,756$, $p=0,000)$, quimioterapia adyuvante $(C=$ $0,438, p=0,036)$, inmunoterapia adyuvante $(C=0,449, p=0,022)$, y antecedentes oncológicos familiares $(C=0,856, p=$ $0,005)$. El estilo de afrontamiento focalizado en la emoción se encontró asociado con el estado civil $(C=0,685, p=0,010)$, la situación laboral $(C=0,760, p=0,001)$, el nivel de estudios alcanzado $(C=0,709$, $p=0,001)$, el tipo de cirugía $(C=0,656$, $p=0,003)$, y antecedentes oncológicos familiares $(C=0,865, p=0,011)$, mientras que el estilo de afrontamiento por evitación se encontró asociado únicamente con el nivel de estudios alcanzado $(C=0,755$, $p=0,000$ ).

\section{El efecto del tipo de tratamiento en los indicadores psicológicos y de CVRS}

Para examinar el efecto del tipo de tratamiento quirúrgico recibido sobre los indicadores psicológicos y de CVRS, se condujeron ANOVAs. En la Tabla 5 se presentan los resultados detallados que reflejan diferencias estadísticamente significativas sobre el nivel de depresión, el estrés percibido, y calidad de vida con respecto al tipo de tratamiento quirúrgico recibido. 


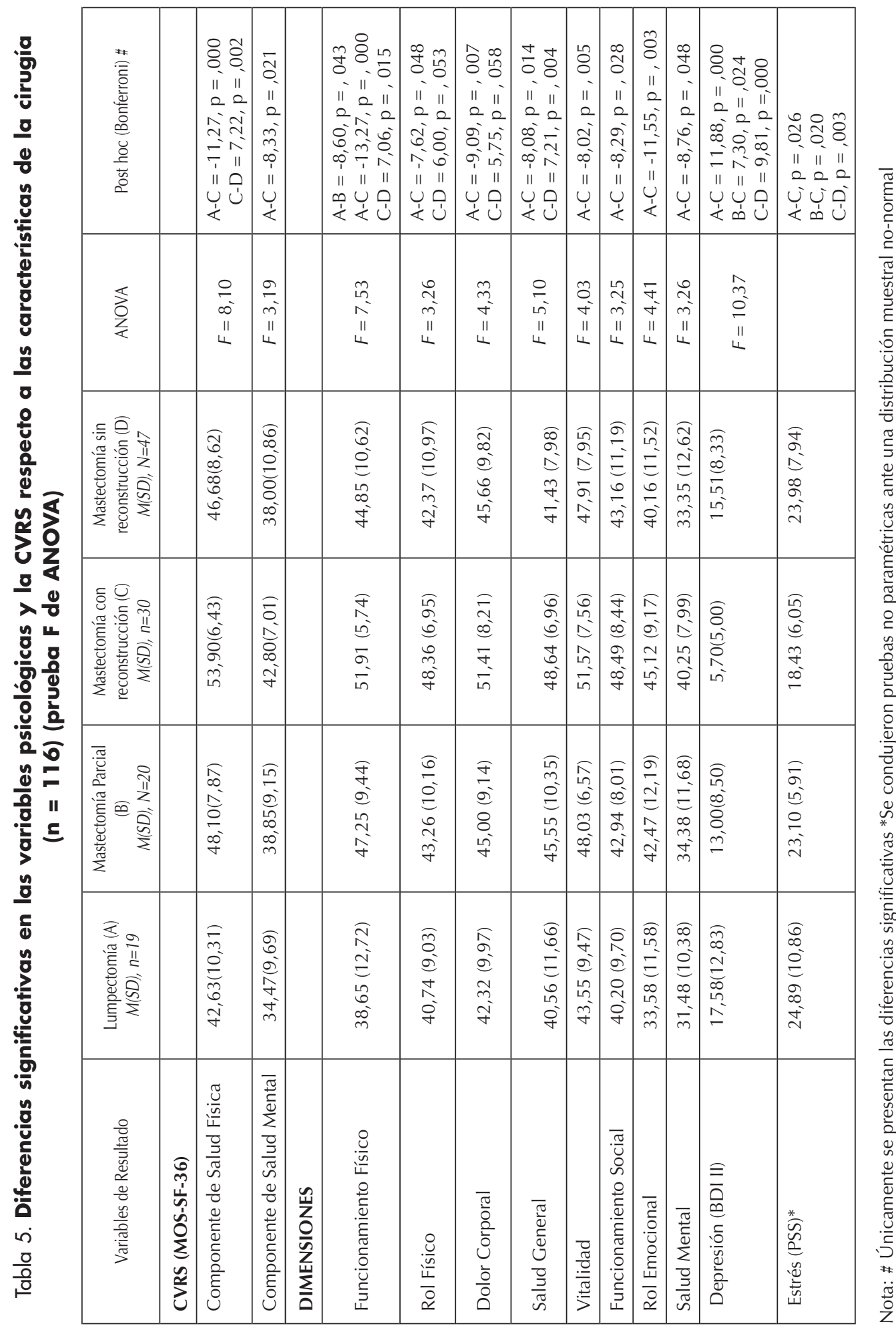




\section{DISCUSIÓN}

Consideramos que una de las fortalezas del presente estudio fue la capacidad para evaluar un número relevante de mujeres con cáncer de mama con variadas características, y en el contexto de Uruguay, pues según nuestro conocimiento, hasta la fecha de la realización del presente estudio, no se ha publicado ningún trabajo con estas características. Por ejemplo, las mujeres que participaron de este estudio eran mayoritariamente mayores de 40 años, residían en Montevideo, se encontraban casadas, conviviendo con hijos, laboralmente activas, y habían alcanzado un nivel de estudios de bachillerato, técnicos, o terciarios. Asimismo, la mayor proporción de estas mujeres habían sido diagnosticadas con cáncer de mama en estadios iniciales (I ó II), 2-5 años previos al momento del estudio, y se encontraba en remisión completa. El tipo de cirugía más frecuente entre las participantes fue mastectomía radical modificada sin reconstrucción, y casi el total de mujeres había recibido quimioterapia, radioterapia, o la combinación de ambos tratamientos adyuvantes. Por otro lado, más de la mitad de participantes había recibido tratamiento psicológico por dificultades vinculadas a la enfermedad o sus tratamientos.

Los resultados de este estudio indicaron que el tipo de cirugía recibida se encontró asociado a diferencias significativas en la CVRS de las participantes. Estos resultados son coincidentes con parte importante de los encontrados en diversos estudios a nivel internacional, algunos de los cuales se refieren en el presente trabajo. Los niveles más elevados de CVRS en los Componentes de Salud Físico y Mental fueron los informados por las mujeres a quienes se les había practicado mastectomía con posterior reconstrucción, mientras que las mujeres a quienes se les practicó una lumpectomía presentaron los niveles más bajos de calidad de vida. Específicamente, se ha considerado en los análisis cada una de las subescalas que componen el MOS-SF-36. Los resultados indicaron que las mujeres a quienes se les había practicado mastectomía con posterior reconstrucción mamaria, informaron de niveles significativamente mayores de CVRS respecto de aquellas intervenidas por medio de una lumpectomía en las subescalas de Función Física, Rol Físico, Dolor Corporal, Salud General, Vitalidad, Función Social, Rol Emocional, y Salud Mental. Asimismo, las mujeres mastectomizadas y posteriormente reconstruidas presentaron puntuaciones indicando mejor CVRS respecto de aquellas mastectomizadas sin posterior reconstrucción mamaria en las subescalas de Función Física, Rol Físico, Dolor Corporal, y Salud General. Las mujeres que recibieron una mastectomía parcial presentaron puntuaciones indicando mejores niveles de calidad de vida (en Función Física) que aquellas lumpectomizadas. En similar dirección, los resultados indicaron niveles de sintomatología depresiva y estrés percibido significativamente mayores entre las mujeres a quienes se les había practicado lumpectomía, mastectomía parcial, ó mastectomía sin posterior reconstrucción mamaria respecto al grupo de mujeres con mastectomía y posterior reconstrucción mamaria, quienes informaron mayores niveles de salud mental. Resultados similares han sido descritos por Girotto et al. ${ }^{(9)}$. Asimismo, otros estudios encontraron que mujeres mastectomizadas sin posterior reconstrucción informaron de niveles menores de salud mental y una más pobre imagen corporal respecto de aquellas a quienes se les había practicado cirugía conservadora o mastectomía con reconstrucción mamaria ${ }^{(7,14,38-45)}$.

Un estudio previo ${ }^{(46)}$, encontró que el impacto a nivel de la sintomatología psicosocial de la mastectomía difería según la edad de las mujeres, especialmente en el corto plazo. La edad ha sido una de las variables sociodemográficas más ampliamen- 
te documentada en relación a la calidad de vida, siendo informada por las mujeres más jóvenes una peor función social y mayor disrupción en las actividades cotidianas ${ }^{(41}$, 44,47), niveles más bajos de calidad de vida global ${ }^{(48,49)}$, peor calidad de vida global, bienestar emocional, y social ${ }^{(8)}$, función emocional, función social, imagen corporal, y perspectiva respecto del futuro ${ }^{(43)}$, mayor malestar $^{(14)}$, mayor sintomatología asociada a la enfermedad ${ }^{(50)}$, función social, finanzas, y percepción de la propia salud futura ${ }^{(51)}$, y función sexual ${ }^{(44,52)}$.

En el presente estudio, las mujeres más jóvenes y con más educación formal fueron quienes informaron de mejor CVRS física, e hicieron mayor uso de los estilos de afrontamiento focalizado en el problema y en la emoción. Por otro lado, las mujeres más jóvenes fueron quienes habían sido diagnosticadas y recibido tratamiento psicológico más recientemente. Estos resultados pueden entenderse dada la mayor necesidad de tomar decisiones y acción que caracteriza las etapas iniciales de la enfermedad y sus tratamientos. Otras investigaciones han encontrado que el cáncer de mama afectaba el bienestar psicosocial y la CVRS en el corto plazo ${ }^{(53-55)}$.

La edad parece estar asociada a menor utilización de un estilo de afrontamiento focalizado en la emoción. El estilo de afrontamiento focalizado en la emoción se encontró asociado a mayores niveles de estrés y sintomatología emocional más severa. Estos resultados corroboran hallazgos anteriores $^{(17,56-62)}$.

Un mayor tiempo transcurrido desde el diagnóstico de cáncer de mama se encontró significativamente asociado a mayores niveles de CVRS y salud mental. En este sentido, el efecto beneficioso del paso del tiempo en relación al impacto del diagnóstico de la enfermedad y sus tratamientos se observó en los niveles de CVRS en sus Componentes Físico y Mental, en los niveles de sintomatología depresiva, y de estrés percibido. Resultados similares han

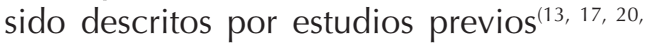
$48,61,63)$.

El estilo de afrontamiento focalizado en el problema (hacer algo para modificar la fuente de estrés), como por ejemplo: aceptación, reinterpretación positiva, y utilización de apoyo social parece hallarse asociado a mejor ajuste. Estudios previos han encontrado una asociación entre estas variables ${ }^{(11,12,13,47)}$, y estilos de afrontamiento $^{(19,64)}$ como predictores de la CVRS de mujeres con cáncer de mama.

En este estudio se replicaron diversos hallazgos de estudios previos en lo que respecta a los correlatos de la CVRS en mujeres con cáncer de mama. Consideramos que nuestros resultados son interesantes, y sugieren pautas potencialmente importantes de cara a la conducción de estudios futuros.

El presente trabajo ha permitido que nos aproximáramos a aspectos del perfil epidemiológico y psicosocial de la población uruguaya de mujeres con cáncer de mama. Los principales resultados obtenidos contribuyen a orientarnos hacia una práctica centrada en la prevención y promoción de salud, conllevando en gran medida esfuerzos y recursos en relación al aún prevalente modelo asistencialista. El impacto psicológico del cáncer de mama en la vida de la paciente es reconocido. Nuestros hallazgos sugieren (y en la dirección de lo informado previamente en la revisión de literatura: Reich y $\left.\operatorname{Remor}^{(65)}\right)$ que las mujeres con cáncer de mama son vulnerables en lo que respecta a su CVRS, y que ésta pueda verse afectada por el malestar psicológico y el desarrollo de la sintomatología depresiva; asimismo, ciertos estilos y estrategias de afrontamiento (p.ej. estilo de afrontamiento focalizado en el problema), y ciertas variables sociodemográficas y laborales (p. ej. haber alcanzado un mayor nivel de estudios formales, encontrarse laboral- 
mente activas), clínicas (p.ej. haber sido diagnosticada en estadios iniciales de la enfermedad, mayor tiempo transcurrido desde el diagnóstico, haber recibido reconstrucción mamaria post-quirúrgica), y psicológicas (p.ej. ausencia de sintomatología de estrés percibido y depresiva) están asociados a un mejor ajuste a la enfermedad y mejor calidad de vida.

Entre las limitaciones más salientes del presente estudio, podemos mencionar el hecho de que la muestra estuvo compuesta predominantemente por mujeres caucásicas, con buen nivel educativo, residentes en la capital de Uruguay, o en la tercer ciudad en densidad poblacional a nivel nacional. Ello indica que los resultados obtenidos podrían diferir en caso de tratarse de una muestra de habitantes de zonas rurales o pequeños pueblos del mismo país. Mayoritariamente, estas mujeres presentaban cáncer no metastásico, y no padecían condiciones de comorbilidad psiquiátrica o médica severas. Esto limita la generalizabilidad de nuestros resultados. Entendemos que el papel del origen étnico, así como el trasfondo cultural y espiritual de las mujeres amerita continuar investigando a fin de establecer su incidencia en el ajuste y CVRS en cáncer de mama.

Definir, reclutar, e identificar la muestra objeto de estudio se encuentra entre los aspectos más complejos en investigación con sobrevivientes de cáncer de mama. Ante esto, consideramos que otra posible limitación de nuestro estudio versa entorno al hecho de que el mismo excluye información de mujeres que hayan declinado la propuesta de participación.

La utilización de un diseño de tipo transversal implica la imposibilidad de obtener evidencia longitudinal, así como de establecer relaciones causales entre factores psicosociales y resultados. El hecho de que no se haya efectuado una evaluación de re-test, limita los alcances de nuestros resultados, dado que no ha sido posible evaluar la estabilidad de los mismos en función del tiempo. Sin embargo, el entendimiento de aquellos aspectos asociados con mejores niveles de ajuste ha permitido aproximarnos a cuestiones que podrían ser abordadas desde las intervenciones.

El muestreo por conveniencia utilizado en este estudio, por medio del cual se reclutó a las participantes en las salas de espera de consulta clínica de seguimiento, y parte de ellas a partir de grupos de apoyo o auto-ayuda, podría derivar en sobreestimar, o bien subestimar el impacto a largo plazo de la enfermedad y sus tratamientos, dado que la participación en este tipo de grupos podría estar relacionada a la experiencia que cada mujer haya tenido con la enfermedad y la supervivencia. Lo antedicho, inevitablemente conlleva también la exclusión de aquellas pacientes que, pese a los beneficios significativos para la morbilidad y mortalidad que ello a menudo implica, no asistan a tales consultas de seguimiento o sesiones de tratamiento.

Otro problema, ampliamente presente también en la literatura en el tema, es que la mayoría de participantes había sido diagnosticada en estadios tempranos. La investigación excluyó a mujeres con discapacidades cognitivas, comorbilidad de enfermedades físicas o psiquiátricas, y en períodos de recrudecimiento de la enfermedad. Esta composición muestral puede sesgar los resultados al incluir aquellas mujeres menos severamente enfermas $y$ tal vez, mejor ajustadas.

A modo de conclusión, y más allá de las limitaciones del presente estudio, cabe destacar que el mismo provee información original e interesante respecto a las variables intervinientes en el ajuste y CVRS de las mujeres uruguayas con cáncer de mama, entre las que cabría señalar la importancia del tiempo trascurrido desde el diagnóstico (tiempo de adaptación), la reconstrucción mamaria, el uso de un estilo de afrontamiento focalizado en el 
problema, la ausencia de estado de ánimo depresivo y de estrés percibido, como factores de protección para la experiencia de una buena calidad de vida en mujeres viviendo con cáncer de mama.

\section{REFERENCIAS BIBLIOGRÁFICAS}

1. Rodríguez SA, Capurso M. Epidemiología del cáncer de mama. AMGO 2006;74: 593-85.

2. Breast Health Global Initiative. Normas internacionales para la salud de la mama y el control de cáncer de mama. Breast J [En línea] 2006 [Acceso 28 de diciembre, 2010] Disponible en: http://www.paho.org/ Spanish/AD/DPC/NC/pcc-breast-cancerguidelines.htm

3. National Cancer Institute. Lo que usted necesita saber sobre el Cáncer de Seno. 23 de abril [En línea] 2010. [Acceso el 17 de enero, 2011] Disponible en: http://www. cancer.gov/espanol/tipos/necesita-saber/ seno/page9

4. Vasallo J, Barrios E. Actualización Ponderada de los Factores de Riesgo del Cáncer. [En línea] Montevideo: Comisión Honoraria de Lucha contra el Cáncer 2003. [Acceso el 19 de enero, 2011] Disponible en: http://www.urucan.org.uy/ uilayer/ve/ factores_riesgo / factores_riesgo _26. pdf

5. Comisión Honoraria de Lucha Contra el Cáncer. Informe Anual [En línea] 2008. [Acceso el 17 de junio 2009] Disponible en: http://www.urucan.org.uy/uilayer/informe_anual.htm

6. Vázquez T, Krygier G, Barrios E, Cataldi S, Vázquez A, Alonso R, et al. Análisis de sobrevida de una población con cáncer de mama y su relación con factores pronósticos: estudio de 1.311 pacientes seguidas durante 230 meses. Rev Med Uruguay 2005;21: 107-21.

7. Wapnir IL, Cody RP, Greco RS. Subtle differences in quality of life after breast cancer surgery. Ann Surg Oncol 1999; 6: 359-66. Doi:10.1007/s10434-999-0359-y
8. Bulotienë G, Ostapenko V, Veseliunas J. Influence of social factors on the quality of life after breast cancer surgical treatment. Acta Médica Lituana 2005;12 (2): 79-83.

9. Girotto JA, Schreiber J, Nahabedian MY. Breast reconstruction in the elderly: Preserving excellent quality of life. Ann Plas Surg 2003; 50 (6): 572-8. Doi: 10.1097/01. SAP.0000069064.68579.19

10. Holland J. History of psycho-oncology: Overcoming attitudinal and conceptual barriers. Psychosom Med 2002;64: 206-21.

11. Deshields T, Tibbs T, Fan M, Taylor M. Differences in patterns of depression after treatment for breast cancer. Psychooncology 2006;15:398-406. Doi:10.1002/ pon.962

12. So WK, Marsh G, Ling WM, Leung FY, Lo JC, Yeung, M, et al. Anxiety, depression and quality of life among chinese breast cancer patients during adjuvant therapy. Eur J Oncol Nurs 2010; 14:17-22. Doi:10.1016/j. ejon.2009.07.005

13. Yen J, Ko C, Yen C, Yang C, Wu C, Juan $\mathrm{C}$, et al. Quality of life, depression, and stress in breast cancer women outpatients receiving active therapy in Taiwan. Psychiatry Clin Neurosci 2006; 60:147-53. Doi:10.1111/j.1440-1819.2006.01479.x

14. Costanzo ES, Lutgendorf SK, Mattes ML, Trehan S, Robinson CB, Tewfik F, et al. Adjusting to life after treatment: distress and quality of life following treatment for breast cancer. Br J Cancer 2007; 97: 1625-31.

15. Golden-Kreutz DM, Andersen BL. Depressive symptoms after breast cancer surgery: Relationships with global, cancer-related, and life event stress. Psychooncology 2004;13: 211-20. Doi:10.1002/pon.736

16. Carver CS, Pozo C, Harris SD, Noriega V, Scheier MF, Robinson DS, et al. How coping mediates the effect of optimism on distress: A study of women with early stage breast cancer. J Pers Soc Psychol 1993; 65: 375-90. Doi:10.1037/0022-3514.65.2.375

17. Osowiecki DM, Compas BE. A prospective study of coping, perceived control, and 
psychological adaptation to breast cancer. Cognitive Ther Res 1999; 23 (2): 169-80. Doi: 10.1023/A:1018779228432

18. Pieterse K, Van Dooren S, Seynaeve C, Bartels CC, Rijnsburger AJ, De Koning HJ, et al. Passive coping and psychological distress in women adhering to regular breast cancer surveillance. Psychooncology 2007;16: 851-8. Doi:10.1002/pon.1135

19. Kershaw T, Northouse L, Kritpracha C, Schafenacker A, Mood D. Coping strategies and quality of life in women with advanced breast cancer and their family caregivers. Psychol Health 2004; 19 (2):139-55. Doi:1 0.1080/08870440310001652687

20. Culver JL, Arena PL, Antoni MH, Carver CS. Coping and distress among women under treatment for early stage breast cancer: Comparing African Americans, Hispanics, and non-Hispanic Whites. Psychooncology 2002;11:495-504. Doi:10.1002/pon.615

21. Perry S, Kowalski TL, Chang CH. Quality of life assessment in women with breast cancer: benefits, acceptability and utilization. Health Qual Life Outcomes 2007;5(24):114. Doi: 10.1186/1477-7525-5-24

22. Epping-Jordan JE, Compas BE, Osowiecki DM, Oppedisano G, Gerhardt CA, Primo K, Krag DN. Psychological adjustment to breast cancer: Processes of emotional distress. Health Psychol 1999; 18:1-12. Doi:10.1037/0278-6133.18.4.315

23. Hack TF, Degner LF. Coping responses following breast cancer diagnosis predict psychological adjustment three years later. Psychooncology 2004; 13: 235-47. Doi:10.1002/pon.739

24. Ganz PA, Kwan L, Stanton AL, Krupnick JL, Rowland JH, Meyerowitz BE, et al. Quality of life at the end of primary treatment of breast cancer: first results from the moving beyond cancer randomized trial. J Natl Cancer I 2004; 96(5):376-87. Doi:10.1093/ jnci/djh060

25. Glanz K, Lerman C. Psychosocial impact of breast cancer: A critical review. Ann Behav Med 1998; 14: 204-12.
26. Moyer A, Salovey P. Psychosocial sequelae of breast cancer and its treatment. Ann Behav Med 1996; 18: 110-25. Doi:10.1007/ BF02909583

27. Carver CS, Smith RG, Petronis VM, Antoni $\mathrm{MH}$. Quality of life among long term survivors of breast cancer: different types of antecedents predict different classes of outcomes. Psychooncology 2006;15:74958. Doi:10.1002/pon.1006

28. National Cancer Institute. Cancer Basics. 20 de febrero, 2009 [Acceso el 31 de marzo 2010] Disponible en: http://www.cancer.gov/cancertopics/what-is-cancer

29. Ware JE, Sherbourne CD. The MOS 36-item short-form health survey. (SF-36): Conceptual framework and item selection. Med Care 1992;30:473-83.

30. Ware JE. SF-36 Health Survey Update. Spine 2000;25:3130-39. Doi:10.1097/00007632200012150-00008

31. Beck AT, Steer R, Brown G. Inventario de Depresión de Beck: BDI II ( $1^{\text {a }}$ ed.). Buenos Aires: Paidós, 2006

32. Penley JA, Wiebe JS, Nwosu A. Psychometric roperties of the Spanish Beck Depression Inventory-II in a medical sample. Psychol Assess 2003; 15(4):569-77. Doi: 10.1037/1040-3590.15.4.569

33. Carver CS. You want to measure coping but your protocol's too long: Consider the Brief COPE. Int J Behav Med 1997;4:92100. Doi:10.1207/s15327558ijbm0401_6

34. Perczek R, Carver CS, Price AA, PozoKaderman C. Coping, mood, and aspects of personality in spanish translation and evidence of convergence with English versions 2000. University of Miami, Department of Psychology [Acceso el 18 de mayo de 2009] Disponible en: http://www. psy.miami.edu/faculty/ccarver/sclspan.html

35. Cohen S, Kamarck T, Mermelstein R. A global measure of perceived stress. J Health Soc Behav 1983; 24:385-96. Doi:10.2307/2136404

36. Remor E. Psychometric properties of a european spanish version of the Perceived 
Stress scale (PSS). Span J Psychol 2006;9 (1):86-93.

37. León OG, Montero I. Métodos de investigación en psicología y educación ( $3^{\mathrm{a}}$ edición). McGraw-Hill: Madrid, 2003

38. Arraras JI, Martínez M, Manterota A, Laínez N. La evaluación de la Calidad de Vida del paciente oncológico. El grupo de Calidad de Vida de la EORTC. Psicooncología 2004; 1 (1): 87-98.

39. Curran D, Van Dongen J, Aaronson NK, Kiebert G, Fentiman IS, Mignolet $F$ et al. Quality of Life of early-stage breast cancer patients treated with radical mastectomy or breast conserving procedures: Results of the EORTC trial 10801. Eur J Cancer 1998; 34: 307-14. Doi:10.1016/S09598049(97)00312-2

40. de Haes JCJ, Curran D, Aaronson NK, Fentiman IS, and On behalf of the European Organisation for Research and Treatment of Cancer (EORTC) Breast Cancer Cooperative Group (BCCG). Quality of life in breast cancer patients aged over 70 years, participating in the EORTC 10850 randomized clinical trial. Eur J Cancer 2003; 39 (7): 94551. Doi: 10.1016/S0959-8049(03)00149-7

41. Engel J, Kerr J, Schlesinger-Raab A, Eckel R, Sauer H, Hölzel D. Predictors of quality of life of breast cancer patients. Acta Oncol 2003; 42(7):710-18. Doi:10.1080/02841860310017658

42. Janni W, Rjosk D, Dimpfl TH, Haertl K, Strobl B, Hepp F et al. Quality of life influenced by primary surgical treatment for stage I-III breast cancer-long-term followup of a matched-pair analysis. Ann Surg Oncol 2001; 8(6): 542-8. Doi:10.1245/ aso.2001.8.6.542

43. Janz NK, Mujahid M, Lantz PM, FagerlinA, Salem B, Morrow M, et al. Populationbased study of the relationship of treatment and sociodemographics on quality of life for early stage breast cancer. Qual Life Res: 2005; 14: 1467-79. Doi:10.1007/s11136005-0288-6

44. Schou I, Ekeberg O, Sandvik L, Hjermstad $\mathrm{M} \mathrm{J}$, Ruland CM. Multiple predictors of health-related quality of life in early stage breast cancer. Data from a year follow-up study compared with the general population. Qual Life Res 2005; 14: 1813-23. Doi:10.1007/s11136-005-4344-z

45. Suárez GL, Rueda AN. Prevalencia e trastorno depresivo mayor en pacientes con mastectomía radical modificada sin reconstrucción. MedUNAB 2007; 10(1): 24-7.

46. Maunsell E, Brisson J, Deschenes L. Psychological distress after initial treatment for breast cancer: a comparison of partial and total mastectomy. J Clin Epidemiol 1989; 42: 765-71. Doi:10.1016/08954356(89)90074-7

47. Shapiro S, López A, Schwartz G, Bootzin R, Figueredo A, Braden C, et al. Quality of life and breast cancer: Relationship to psychosocial variables. J Clin Psychol 2001; 57(4):501-19. Doi:10.1002/jclp.1026

48. King MT, Kenny P, Shiell A, Hall J, Boyages J. Quality of life three months and one year after first treatment for early stage breast cancer: Influence of treatment and patient characteristics. Qual Life Res 2000;9: 789800. Doi:10.1023/A:1008936830764

49. Vacek PM, Winstead-Fry P, Secker-Walker $\mathrm{RH}$, Hooper GJ, Plante DA. Factors influencing quality of life in breast cancer survivors. Qual Life Res 2003; 12: 527-37. Doi:10.1023/A:1025098108717

50. Janz NK, Mujahid M, Chung LK, Lantz PM, Hawley ST, Morrow M, et al. Symptom experience and quality of life of women following breast cancer treatment. J Womens Health 2007; 16(9):1348-61. Doi:10.1089/ jwh.2006.0255

51. Engel J, Kerr J, Schlesinger-Raab A, Sauer J, Hölzel D. Quality of life following breastconserving therapy or mastectomy: Results of a 5-year prospective study. Breast J 2004; 10 (3) :223-31. Doi:10.1111/j.1075122X.2004.21323.X

52. Casso D, Buist D, Taplin S. Quality of life of 5-10 year breast cancer survivors diagnosed between age 40 and 49. Health Qual Life Outcomes 2004; (25):1-9. Doi:10.1186/1477-7525-2-25 
53. Aragona M, Muscatello MR, Mesiti M. Depressive mood disorders in patients with operable breast cancer. J Exp Clin Canc Res 1997;16:111-18.

54. Kornblith AB, Herndon JE, Weiss RB, Zhang C, Zuckerman EL, Rosenberg S, et al. Longterm adjustment of survivors of early-stage breast carcinoma, 20 years after adjuvant chemotherapy. Cancer 2003; 98:679-89. Doi:10.1002/cncr.11531

55. Zabora J, Brintzenhofeszoc K, Curbow B, Hooker C, Piantadosi S. The prevalence of psychological distress by cancer site. Psychooncology 2001; 10:19-28. Doi:10.1002/1099$1611(200101 / 02) 10: 1<19:$ : AID PON501>3.0.CO;2-6

56. Bishop SR, Warr D. Coping, catastrophizing and chronic pain in breast cancer. J Behav Med 2003; 26 (3):265-81. Doi:10.1023/A:1023464621554

57. Cohen M. Coping and emotional distress in primary and recurrent breast cancer patients. J Clin Psychol Med S 2002; 9(3):24551.

58. Gilbar O. Breast cancer: how do Israeli women cope? A cross-sectional sample. Fam Syst Health 2005; 23(2):161-71. Doi:10.1037/1091-7527.23.2.161

59. Gilbar O, Hevroni A. Counterfactuals, coping strategies, and psychological distress among breast cancer patients. Anxiety
Stress Coping 2007; 20 (4): 383-92. Doi: 10.1080/10615800701384439

60. Iwamitsu I, Shimoda K, Abe H, Tani T, Okawa M, Buck R. Anxiety, emotional suppression, and psychological distress before and after breast cancer diagnosis. Psychosomatics 2005; 46:19-24. Doi:10.1176/appi. psy.46.1.19

61. Roussi P, Krikeli V, Hatzidimitriou C, Koutri I. Patterns of coping, flexibility in coping and psychological distress in women diagnosed with breast cancer. Cognitive Ther Res 2007;31:97-109. Doi:10.1007/s10608006-9110-1

62. Thomas SF, Marks DF. The measurement of coping in breast cancer patients. Psychooncology 1995;4:231-7. Doi:10.1002/ pon.2960040309

63. Bulotienë G, Ostapenko V, Veseliunas J. Psychological adaptation of breast cancer patients. Acta Medica Lituanica 2006;13 (2): 92-6.

64. Holland K, Holahan CK. The relation of social support and coping to positive adaptation to breast cancer. Psychol Health 2003;18 (1):15-29. Doi: 10.1080/0887044031000080656

65. Reich M, Remor E. Variables psicosociales asociadas con la calidad de vida relacionada con la salud en mujeres con cáncer de mama post-cirugía: una revisión sistemática. Cien Psicol 2010; 4(2):179-223. 